\title{
POLÍTICAS PÚBLICAS E DESENVOLVIMENTO HUMANO: FATORES QUE IMPACTAM O IDH EM MUNICÍPIOS BRASILEIROS
}

\author{
Public policies and human development: factors that impact the HDI in \\ brazilian municipalities
}

Flavio Saab

E-mail: flaviosaab@gmail.com

Mestre e Doutorando em Administração pela Universidade de Brasília - PPGA/UnB; Professor Colaborador do Departamento de Gestão de Políticas Públicas da Universidade de Brasília - GPP/ UnB.

Endereço para contato: Universidade de Brasília - UnB, Campus Darcy Ribeiro, Faculdade de Administração, Contabilidade, Economia e Gestão de Políticas Públicas-FACE, Asa Norte, Brasília-

DF, Brasil, Cep 70910-900. https://orcid.org/0000-0002-3913-4175

Fagner de Oliveira Dias E-mail: fagner.pmdf@gmail.com

Mestre e Doutorando em Administração pela Universidade de Brasília - PPGA/UnB; Professor Colaborador do Departamento de Administração da Universidade de Brasília - ADM/UnB. Endereço para contato: Universidade de Brasília - UnB, Campus Darcy Ribeiro, Faculdade de Administração, Contabilidade, Economia e Gestão de Políticas Públicas-FACE, Asa Norte, Brasília-

DF, Brasil, Cep 70910-900. https://orcid.org/0000-0001-6529-5485

André Vaz Lopes

E-mail: andrevazlopes@gmail.com

Doutorando em Administração pela Universidade de Brasília - UnB.

Endereço para contato: Universidade de Brasília - UnB, Campus Darcy Ribeiro, Faculdade de Administração, Contabilidade, Economia e Gestão de Políticas Públicas-FACE, Asa Norte, Brasília-

DF, Brasil, Cep 70910-900. https://orcid.org/0000-0002-8929-2374

Pedro Ivo Sebba Ramalho

E-mail: pedroivosr@gmail.com

Doutor em Ciências Sociais pela Universidade de Brasília - UnB;

Pesquisador do Centro de Estudos Sociais na Universidade de Coimbra - CES/UC.

Endereço para contato: Colégio de S. Jerônimo, Apartado 3087, 3000-995, Coimbra, Portugal. https://orcid.org/0000-0002-1710-2500

Artigo recebido em 28 de outubro de 2019. Aceito em 26 de agosto de 2021. 


\section{RESUMO}

A abordagem do desenvolvimento humano considera que apenas o crescimento econômico não é suficiente para explicar o progresso, que decorre de um processo maior de transformação. Neste contexto, o Índice de Desenvolvimento Humano - IDH tem se mostrado um importante indicador para apoiar o direcionamento de políticas públicas capazes de promover o progresso e aumentar a qualidade de vida das populações. Para contribuir com este debate, a presente pesquisa tem como objetivo investigar quais fatores explicam o IDH de municípios brasileiros. A partir da literatura, foram identificadas variáveis socioeconômicas com potencial relação funcional com o IDH. Por meio de análise de dados em painel de 1.292 municípios brasileiros, selecionados por posição geográfica, representatividade populacional e pela disponibilidade de dados, foram testadas as três formulações econométricas alternativas: Pooled Regression, Modelo de Efeitos Aleatórios e Modelo de Efeitos Fixos. Os resultados da regressão indicaram que as condições de energia elétrica, tamanho populacional, localização, renda e saneamento se mostraram fatores explicativos do IDH. Do ponto de vista teórico, os resultados complementaram estudos que relacionam variáveis socioeconômicas ao IDH e, em perspectiva prática, indicou caminhos para que políticos e gestores possam promover políticas públicas capazes de melhorar o desenvolvimento humano em municípios brasileiros. Palavras-chave: Políticas públicas, Desenvolvimento humano, Índice de Desenvolvimento Humano.

\section{ABSTRACT}

The human development approach considers that economic growth alone is not sufficient to explain progress, which results from a greater process of transformation. In this context, the Human Development Index - HDI has proved to be an important indicator to support the direction of public policies capable of promoting progress and increasing the quality of life of the populations. To contribute to this debate, the present research aims to investigate which factors explain the HDI of Brazilian municipalities. From the literature, socioeconomic variables with potential functional relationship with the HDI were identified. Through panel data analysis of 1,292 Brazilian municipalities, selected by geographical position, population representativeness and data availability, the three alternative econometric formulations were tested: Pooled Regression, Random Effects Model and Fixed Effects Model. The results of the regression indicated that the conditions of electricity, population size, location, income and sanitation proved to be explanatory factors for the HDI. From a theoretical point of view, the results complemented studies that relate socioeconomic variables to the HDI and, in a practical perspective, indicated ways for politicians and managers to promote public policies capable of improving human development in Brazilian municipalities.

Keywords: Public Policies; Human Development; Human Development Index.

\section{INTRODUÇÃO}

As políticas públicas representam a transformação das propostas e ideias dos governos em ações e programas que impactam o mundo real (Souza, 2006). Pode-se compreender a política pública como a implantação de um projeto de governo, sob a responsabilidade do Estado (Höfling, 2001), na busca por ações que influenciem determinadas questões da sociedade (Dias \& Matos, 2012). O objetivo das autoridades governamentais é estabelecer políticas públicas de suas preferências e bloquear aquelas que lhes sejam inconvenientes, em 
processo de escolha que deve buscar o tratamento ou a solução de problemas públicos (Dias \& Matos, 2012; Secchi, 2015). Quando o governo escolhe políticas adequadas, ele atende às necessidades da população e oferece real contribuição para a melhoria na vida das pessoas (Mattei et al., 2018).

Neste aspecto, o Índice de Desenvolvimento Humano - IDH tem se mostrado um importante indicador para apoiar o direcionamento de políticas públicas. Criado em meados de 1990, pela Organização das Nações Unidas - ONU, o IDH objetiva verificar o grau de desenvolvimento de países, regiões, estados e municípios, a partir de indicadores de desempenho pré-estabelecidos e relacionados a saúde, educação e renda (Scarpin \& Slomski, 2007). Assim, a partir da difusão do uso do IDH, as autoridades públicas puderam orientar políticas públicas de modo a buscar desenvolvimento não apenas por meio de aumento da riqueza, mas também por meio de melhoria nos níveis de saúde, serviços médicos, educação, condições de trabalho, direitos, liberdades, relações familiares e infraestrutura (Angelo et al., 2009; Scarpin \& Slomski, 2007).

A difusão do uso do IDH e sua aplicação na área de políticas públicas deu importante impulso para pesquisas na área (Guimarães \& Jannuzzi, 2005) e tem propiciado a identificação de fatores determinantes para o aumento da qualidade de vida das populações. Neste contexto, alguns estudos têm sido desenvolvidos e têm contribuído para a compreensão das variáveis socioeconômicas que se relacionam com o IDH. Estudos realizados no Brasil buscaram analisar fatores explicativos do desenvolvimento humano em determinados contextos do país (Angelo et al., 2009; Bispo, 2009; Matta et al., 2016; Mattei et al., 2018; Scarpin \& Slomski, 2007). Além disso, pesquisas realizadas em outros países buscaram identificar como políticas públicas podem afetar positivamente o IDH (Fadilah et al., 2018; ljaz, 2018; Jalil \& Kamaruddin, 2018; Lestari \& Sanar, 2018; Marinho \& Neto, 1999). A partir disso, identificou-se lacuna na literatura que oportuniza a realização de estudo com maior abrangência territorial e que considere séries temporais em sua análise, como proposto por ljaz (2018) e Mattei et al. (2018).

Assim, a partir desta lacuna, a presente pesquisa pretende alcançar o objetivo de investigar quais fatores explicam o IDH de municípios brasileiros. Para isso, serão analisados os dados de todos os municípios dos Estados de São Paulo, Pernambuco, Paraná, Amazonas e Distrito Federal nos anos de 1991, 2000 e 2010.

O trabalho está dividido em cinco partes, além desta Introdução. Inicialmente, apresenta-se um referencial teórico sobre o tema. Em seguida, apresentam-se seções com os procedimentos metodológicos utilizados, os resultados obtidos e as discussões realizadas. Por fim, as conclusões da pesquisa são apresentadas. 


\section{REFERENCIAL TEÓRICO}

\subsection{O DESENVOLVIMENTO HUMANO E O IDH}

O desenvolvimento é uma construção política da sociedade, caracterizada pela melhoria na qualidade de vida, avanço tecnológico, aprimoramento das instituições e evolução dos indicadores sociais (Castelar, 2009; Sicsú, 2009). O conceito de desenvolvimento, portanto, vai além das definições restritas ao crescimento econômico e deve envolver aspectos relacionados às dimensões sociais e humanas (Khair, 2009).

A abordagem do desenvolvimento humano considera que apenas o crescimento econômico não é suficiente para explicar o progresso dos países (Bilbao-Ubillos, 2013). O progresso decorre de um processo de transformação política, econômica e social, onde o crescimento em apenas uma dessas áreas, isoladamente, consistirá em um desenvolvimento superficial (Bresser-Pereira, 2003). A nova abordagem aponta para o deslocamento da ênfase em agregados econômicos para um foco maior em atender as necessidades humanas básicas, gerar empregos produtivos e reduzir a pobreza (Machado \& Pamplona, 2008).

Nesse sentido, mostrou-se fundamental a contribuição intelectual e a nova perspectiva da dimensão humana do desenvolvimento apresentada pelo Programa das Nações Unidas para o Desenvolvimento - PNUD - agência líder da rede global de desenvolvimento da ONU - em meados de 1990 (Machado \& Pamplona, 2008). Em seu primeiro relatório de desenvolvimento humano, o PNUD apontou o desenvolvimento como elemento mais amplo do que apenas uma medida de riqueza (Sagar \& Najam, 1998):

\footnotetext{
O desenvolvimento humano é um processo de ampliar as escolhas das pessoas. Os mais críticos são levar uma vida longa e saudável, ser educados e desfrutar de um padrão de vida decente. Escolhas adicionais incluem liberdade política, direitos humanos garantidos e respeito (PNUD, 1990).
}

Assim, a partir de 1990, a compreensão do desenvolvimento humano passou a considerar dois aspectos. $\bigcirc$ primeiro relaciona-se com a formação de capacidades humanas, tais como melhoria da saúde, conhecimento e habilidades. $O$ segundo aspecto envolve o uso que as pessoas fazem de suas capacidades adquiridas para fins de lazer, trabalho ou para serem atuantes em assuntos culturais, sociais e políticos (PNUD, 1990).

Com o fortalecimento do conceito de desenvolvimento humano, o uso de indicadores relacionados apenas à renda foi compreendido como insuficiente para a tomada de decisão (Neumayer, 2001; Trabold-Nübler, 1991). Havia a necessidade de suprir deficiências em indicadores de natureza mais restrita e simples, como o Produto Interno Bruno - PIB 
(Guimarães \& Jannuzzi, 2005). O reconhecimento desta necessidade levou o PNUD a desenvolver o IDH, que passou a ser um dos indicadores mais utilizados no planejamento das políticas públicas e avaliação do progresso dos países (Oliveira, 2013).

O IDH foi construído a partir de indicadores-chave. Ainda que o PNUD reconhecesse como desejável que se considerasse muitos indicadores, a instituição optou por dar ênfase a três dimensões essenciais: longevidade, conhecimento e padrão de vida. A ênfase nestas dimensões visava ajudar os formuladores de políticas a compreenderem o que é realmente crucial para o desenvolvimento (PNUD, 1990).

Na dimensão da longevidade, o indicador adotado é a esperança de vida ao nascer, tendo em vista o entendimento de que uma vida longa é valiosa e que uma nutrição adequada e boa saúde estão intimamente associados à maior expectativa de vida. Na dimensão do conhecimento, a alfabetização e os níveis de escolaridade são enfatizados. Na dimensão do padrão de vida, faz-se o uso de um indicador de renda. O Quadro 1 apresenta o IDH e os indicadores que o compõe.

Quadro 1

Composição do IDH

\begin{tabular}{|c|c|c|c|}
\hline Dimensão & Índices & & Cálculo \\
\hline Longevidade $\left(\mathrm{I}_{\text {vida }}\right)$ & $\begin{array}{l}\text { - Expectativa de Vida } \\
\text { ao Nascer (EVN) }\end{array}$ & $\mathrm{I}_{\text {vida }}=$ & $\begin{array}{l}\text { EVN } \quad \text { - valor mínimo } \\
\text { valor máximo - valor mínimo }\end{array}$ \\
\hline Conhecimento $\left(I_{\text {educą̧ão }}\right)$ & $\begin{array}{l}\text { - Anos Médios de Estu- } \\
\text { do (AME) } \\
\text { - Anos Esperados de } \\
\text { Escolaridade (AEE) }\end{array}$ & $\mathrm{I}_{\text {educação }}=$ & - $\frac{\sqrt{A M E x A E E} \text { - valor mínimo }}{\text { valor máximo - valor mínimo }}$ \\
\hline Padrão de Vida $\left(I_{\text {renda }}\right)$ & $\begin{array}{l}\text { - Renda Per Capta } \\
\text { (RPC) }\end{array}$ & $\mathrm{I}_{\text {renda }}=$ & $\frac{\ln (\mathrm{RPC})}{\ln (\text { valor máximo) }-\ln (\text { valor mínimo })}$ \\
\hline
\end{tabular}

Fonte: Elaboração própria a partir de PNUD (2010).

De acordo com o PNUD (2010), o cálculo do IDH é desenvolvido em duas etapas. Na primeira, ajusta-se os valores mínimos e máximos dos índices $I_{\text {vida' }} I_{\text {educação' }} I_{\text {renda }}$, para transformá-los em índices que variam entre zero (0) e um (1) (Quadro 1). Em seguida, estes índices são agregados de modo a obter-se o IDH (Equação 1):

$$
\mathrm{IDH}=\left(\left.\right|_{\text {vida }}{ }^{1 / 3} \times\left.\right|_{\text {educação }} ^{1 / 3} \times\left.\right|_{\text {rendimento }} ^{1 / 3}\right)
$$

A aplicação da Equação 1 em diferentes contextos apresentará níveis de IDH que variam entre zero (0) e um (1). Para facilitar a compreensão, o PNUD (2010) criou parâmetros para enquadramento dos países em faixas que representam níveis de desenvolvimento humano, de acordo com o IDH medido (Quadro 2). 
Quadro 2

Composição do IDH

\begin{tabular}{ll}
\hline Faixa & Nível do Desenvolvimento Humano \\
\hline $\mathrm{IDH}<0,488$ & Baixo \\
\hline $0,488 \leq \mathrm{IDH}<0,677$ & Médio \\
\hline $0,677 \leq \mathrm{IDH}<0,788$ & Elevado \\
\hline $\mathrm{IDH} \geq 0,788$ & Muito elevado \\
\hline
\end{tabular}

Fonte: Elaboração própria a partir de PNUD (2010)

A fácil compreensão e aplicação do IDH pode ser considerada o aspecto mais importante para que o índice tenha alcançado resultados significativos desde sua criação. No entanto, sabe-se que ele é incapaz de captar todas as dimensões do desenvolvimento humano, o que fomenta um importante debate entre teóricos sobre como qualificar o índice em perspectiva mais abrangente (Oliveira, 2013). Nesse aspecto, destaca-se uma das discussões que avançam na literatura, a qual envolve a necessidade de incorporação da sustentabilidade ambiental como dimensão do desenvolvimento humano (Martins et al., 2006).

Enquanto a discussão sobre o melhor uso do IDH avança, pesquisadores, cientistas e gestores públicos dedicam esforços contínuos ao aperfeiçoamento do índice e em como utilizá-lo para a tomada de decisão. Para isso, alguns estudos foram desenvolvidos para identificar fatores explicativos do IDH em diversos contextos e realidades. A seção seguinte se propõe a apresentar alguns destes trabalhos.

\subsection{FATORES EXPLICATIVOS DO IDH}

O IDH promoveu um importante impacto no processo de formulação de políticas públicas. As estimativas do IDH dos países, bem como os IDH desagregados por regiões e municípios tiveram o efeito pretendido de concentrar maior atenção nas capacidades humanas básicas e levar os formuladores de políticas de cada localidade a examinar as condições em que se encontrava a população sob sua responsabilidade (Fukuda-Parr, 2003).

Assim, movidos pelas demandas de informação para formulação de políticas e tomadas de decisões nas esferas públicas, instituições de pesquisa e pesquisadores empreenderam esforços para estudar o IDH e seus fatores explicativos. A representação sintética da realidade social proporcionada pelo IDH ajuda a balizar a distribuição de recursos e de políticas públicas (Guimarães \& Jannuzzi, 2005). Neste sentido, alguns estudos têm sido desenvolvidos para identificar quais fatores socioeconômicos possuem relação funcional com - IDH, de modo a permitir a identificação de elementos sobre os quais as políticas públicas podem propiciar maiores níveis de desenvolvimento humano. 
Alguns estudos realizados no Brasil buscaram encontrar fatores condicionantes de melhores níveis de IDH no país. Scarpin e Slomski (2007), por exemplo, identificaram que densidade demográfica, saneamento, distância à capital e população foram explicativos do IDH no Estado do Paraná. Em estudo conduzido por Bispo (2009), em Roraima, o autor encontrou relação funcional entre o IDH e os direitos de propriedade e infraestrutura (água encanada, energia elétrica e telecomunicações). Ainda, Ângelo et al. (2009) identificaram influência de transporte e renda no IDH nos municípios do Estado de Alagoas.

Em dois estudos mais recentes, Matta et al. (2016) e Mattei et al. (2018) analisaram outras relações funcionais entre variáveis socioeconômicas brasileiras e o desenvolvimento humano. No primeiro trabalho, os autores identificaram a atenção primária à saúde, o emprego, a população, o envelhecimento (longevidade), a renda, e os óbitos por causas mal definidas como fatores condicionantes de IDH no Brasil. No segundo trabalho, os autores identificaram que despesas com saúde e educação têm forte influência no IDH de municípios brasileiros.

Em pesquisas realizadas em outros países também é possível verificar importantes achados que relacionam variáveis socioeconômicas com o desenvolvimento humano. ljaz (2018) identificou que renda, infraestrutura, instalações de saúde, sistema educacional, liberdade e saneamento afetam o IDH em 26 países analisados. Os resultados de pesquisa realizada na Indonésia, conduzida por Fadilah et al. (2018), reforçaram a importância de investimento em infraestrutura para alcançar melhores níveis de desenvolvimento humano. Por fim, dois estudos realizados em países em desenvolvimento, indicaram importante relação funcional entre o tempo de escolaridade, expectativa de vida, renda e o IDH (Jalil \& Kamaruddin, 2018; Lestari \& Sanar, 2018).

O quadro 3 apresenta um resumo dos estudos encontrados na literatura e que subsidiam a presente investigação. 
Quadro 3

Resumo das variáveis explicativas do IDH encontradas na literatura

\begin{tabular}{|c|c|c|}
\hline Estudo & $\begin{array}{l}\text { Local da investi- } \\
\text { gação }\end{array}$ & Variáveis explicativas \\
\hline Scarpin e Slomski (2007) & Brasil & $\begin{array}{l}\text { altitude, densidade demográfica, saneamento, distân- } \\
\text { cia à capital e população }\end{array}$ \\
\hline Ângelo et al. (2009) & Brasil & $\begin{array}{l}\text { localização, eletricidade, população, população eco- } \\
\text { nomicamente ativa, renda, transporte, saneamento e } \\
\text { violência }\end{array}$ \\
\hline Bispo (2009) & Brasil & $\begin{array}{l}\text { direitos de propriedade e infraestrutura (água enca- } \\
\text { nada, energia elétrica e telecomunicações) }\end{array}$ \\
\hline Matta et al. (2016) & Brasil & $\begin{array}{l}\text { atenção primária à saúde, emprego, população urba- } \\
\text { na, envelhecimento (longevidade), renda, óbitos por } \\
\text { causa mal definidas }\end{array}$ \\
\hline ljaz (2018) & $\begin{array}{l}26 \text { países em } \\
\text { desenvolvimento }\end{array}$ & $\begin{array}{l}\text { renda, infraestrutura, instalações de saúde, sistema } \\
\text { educacional, liberdade e saneamento }\end{array}$ \\
\hline Fadilah et al. (2018) & Indonésia & infraestrutura \\
\hline Jalil e Kamaruddin (2018) & $\begin{array}{l}15 \text { países em de- } \\
\text { senvolvimento }\end{array}$ & $\begin{array}{l}\text { tempo de estudo, a expectativa de vida e PIB per } \\
\text { capita }\end{array}$ \\
\hline Lestari e Sanar (2018) & Indonésia & tempo de estudo, expectativa de vida e PIB \\
\hline Mattei et al. (2018) & Brasil & despesas com saúde e educação \\
\hline
\end{tabular}

Fonte: Elaboração própria.

Os resultados dessas pesquisas encontradas na literatura contribuem para a identificação de variáveis com potencial de explicação do IDH.

\section{METODOLOGIA}

Nesta seção são apresentadas as fontes de dados, dados da amostra e as variáveis utilizadas no estudo, bem como o modelo econométrico utilizado. Para realizar a análise foi utilizado o pacote estatístico Gretl'.

\subsection{POPULAÇÃO E AMOSTRA}

Foram utilizados os dados de 1.292 municípios brasileiros, localizados em cinco unidades da federação (UF), distribuídas nas cinco regiões do Brasil. A amostra utilizada nesta pesquisa representou $23,5 \%$ dos municípios brasileiros e $34,64 \%$ da população total do País (Tabela 1). 
Tabela 1

Características da Amostra (Dados de 2010)

\begin{tabular}{lllrrrr}
\hline $\begin{array}{c}\text { Unidade da Fe- } \\
\text { deração (UF) }\end{array}$ & Sigla & Região & $\begin{array}{c}\text { Quantidade } \\
\text { de Municípios }\end{array}$ & $\begin{array}{c}\text { \% Municípios } \\
\text { Brasileiros }\end{array}$ & População & $\begin{array}{c}\text { \% População } \\
\text { Brasileira }\end{array}$ \\
\hline Amazonas & AM & Norte & 64 & $1,2 \%$ & 3.483 .686 & $1,83 \%$ \\
\hline Distrito Federal & DF & Centro-Oeste & 1 & $0,0 \%$ & 2.543 .802 & $1,33 \%$ \\
\hline Pernambuco & PE & Nordeste & 181 & $3,3 \%$ & 8.639 .684 & $4,53 \%$ \\
\hline Paraná & PR & Sul & 410 & $7,4 \%$ & 10.617 .540 & $5,57 \%$ \\
\hline São Paulo & SP & Sudeste & 636 & $11,5 \%$ & 40.788 .367 & $21,38 \%$ \\
\hline Total da Amostra & & & 1.292 & $23,5 \%$ & 66.073 .079 & $34,64 \%$ \\
\hline
\end{tabular}

Fonte: Elaboração própria.

A escolha dos municípios pertencentes à amostra obedeceu a quatro critérios: i) foram selecionados todos os municípios das UFs investigadas para se evitar viés de seleção de municípios; ii) foram selecionadas cinco UFs, sendo uma representante de cada região do Brasil, de modo a representar as diferentes realidades socioeconômicas do País; iii) foram selecionadas UFs de elevada representatividade populacional em suas regiões; iv) a disponibilidade de dados públicos orientou a escolha das UFs investigadas em cada região.

\subsection{DEFINIÇÃO DAS VARIÁVEIS E FONTES DOS DADOS}

Este estudo investiga a relação funcional entre variáveis socioeconômicas (variáveis independentes) e o Índice de Desenvolvimento Humano - IDH (variável dependente). A partir da literatura, foram selecionadas seis variáveis socioeconômicas explicativas do IDH e identificada a expectativa teórica da relação entre elas e o IDH. O Quadro 4 indica a descrição dessas variáveis, a literatura de suporte, o sinal esperado e as fontes dos dados utilizadas. 
Quadro 4

Variáveis e Fontes de Dados

\begin{tabular}{|c|c|c|c|c|}
\hline Variável & Descrição & Literatura de Suporte & $\begin{array}{l}\text { Sinal } \\
\text { Espera- } \\
\text { do }\end{array}$ & Fonte dos Dados: \\
\hline $\begin{array}{l}\text { Índice de } \\
\text { Desenvolvimento } \\
\text { Humano (IDH) }\end{array}$ & $\begin{array}{l}\text { Índice de Desenvolvimento } \\
\text { Humano }\end{array}$ & NA & NA & $\begin{array}{l}\text { http://www.atlasbrasil. } \\
\text { org.br }\end{array}$ \\
\hline $\begin{array}{l}\text { Energia Elétrica } \\
\text { (ENE) }\end{array}$ & $\begin{array}{l}\text { \% da população em domi- } \\
\text { cílios com energia elétrica }\end{array}$ & $\begin{array}{l}\text { ljaz (2018) e Fadilah et } \\
\text { al. (2018) }\end{array}$ & + & $\begin{array}{l}\text { http://www.atlasbrasil. } \\
\text { org.br }\end{array}$ \\
\hline $\begin{array}{l}\text { Localização } \\
\text { (LOC) }\end{array}$ & $\begin{array}{l}\text { Distância à capital estadu- } \\
\text { al para os municípios }\end{array}$ & $\begin{array}{l}\text { Scarpin e Slomski } \\
\text { (2007) }\end{array}$ & $+1-$ & $\begin{array}{l}\text { http://www.ipeadata. } \\
\text { gov.br }\end{array}$ \\
\hline $\begin{array}{l}\text { População } \\
\text { (POP) }\end{array}$ & População residente total & $\begin{array}{l}\text { Scarpin e Slomski } \\
\text { (2007) e Matta et al. } \\
\text { (2016) }\end{array}$ & - & $\begin{array}{l}\text { http://www.atlasbrasil. } \\
\text { org.br }\end{array}$ \\
\hline Renda (REN) & Renda per capta & $\begin{array}{l}\text { ljaz (2018), Ângelo et } \\
\text { al. (2009), Matta et al. } \\
\text { (2016), Jalil e Kmarud- } \\
\text { din (2018) e Lestari e } \\
\text { Sanar (2018) }\end{array}$ & + & $\begin{array}{l}\text { http://www.atlasbrasil. } \\
\text { org.br }\end{array}$ \\
\hline $\begin{array}{l}\text { Saneamento } \\
\text { (SAN) }\end{array}$ & $\begin{array}{l}\text { \% da população em domi- } \\
\text { cílios com banheiro e água } \\
\text { encanada }\end{array}$ & $\begin{array}{l}\text { Scarpin e Slomski } \\
\text { (2007), ljaz (2018) e } \\
\text { Fadilah et al. (2018) }\end{array}$ & + & $\begin{array}{l}\text { http://www.atlasbrasil. } \\
\text { org.br }\end{array}$ \\
\hline Violência (VIO) & $\begin{array}{l}\text { Quantidade de homicídios } \\
\text { por município, por } 100 \mathrm{mil} \\
\text { habitantes }\end{array}$ & Matta et al. (2016) & - & $\begin{array}{l}\text { http://www.ipeadata. } \\
\text { gov.br }\end{array}$ \\
\hline
\end{tabular}

Fonte: Elaboração própria. NA = Não se aplica.

Os dados do estudo foram obtidos nas plataformas Atlas Brasil e Ipeadata. A Atlas Brasil é uma ferramenta de divulgação de informações sobre o desenvolvimento humano no Brasil e é produto da parceria entre o Programa das Nações Unidas para o Desenvolvimento - PNUD, o Instituto de Pesquisa Econômica Aplicada - IPEA e a Fundação João Pinheiro FJP (Atlas Brasil, 2021). Já o lpeadata é uma base de dados macroeconômicos, financeiros e regionais do Brasil mantida pelo IPEA (Ipeadata, 2021). Para realização desta pesquisa, foram utilizados os dados dos anos de 1991, 2000 e 2010, nos quais houve medição do IDH, variável dependente do estudo.

\subsection{MODELO ECONOMÉTRICO}

A regressão é um método que pode ser usado para representar relacionamentos entre variáveis independentes e prever um resultado de determinada variável dependente (Gelman \& Hill, 2007). Como na presente pesquisa o objetivo é investigar quais fatores explicam o IDH de municípios brasileiros, a regressão é uma metodologia adequada para relacionar fatores socioeconômicos ao IDH, verificando qual influência esses fatores exercem 
sobre o Índice. Para isso, foi utilizada a regressão linear múltipla no trabalho (Gelman \& Hill, 2007; Matta et al., 2016).

Conforme já apresentado no Quadro 4, os fatores selecionados para o modelo foram Energia Elétrica (ENE), Localização (LOC), População (POP), Renda (REN), Saneamento (SAN) e Violência (VIO). Para avaliar a relação funcional destes fatores com o IDH, seguindo método proposto por Jalil e Kamaruddin (2018), Matta et al. (2016), Mattei et al. (2018) e Scarpin e Slomski (2007), a modelagem econométrica é apresentada na Equação 2:

$\mathrm{IDH}_{\mathrm{it}}=\beta_{1}+\beta_{2} \mathrm{ENE}_{\mathrm{it}}+\beta_{3} \mathrm{LnLOC}_{\mathrm{it}}+\beta_{4} \mathrm{LnPOP}_{\mathrm{it}}+\beta_{5} \mathrm{REN}_{\mathrm{it}}+\beta_{6} \mathrm{SAN}_{\mathrm{it}}+\beta_{7} \mathrm{VIO}_{\mathrm{it}}+\varepsilon_{\mathrm{it}}$

Na Equação 2, o termo de erro pode ser decomposto em $\varepsilon_{i t}=\mu_{i t}+v_{i t^{\prime}}$ em que $\varepsilon_{i t}$ é o efeito específico individual; $\mu_{i t}$ é o distúrbio restante $e_{;} v_{i t}$ varia ao longo do tempo e das unidades de observação, capturando o que deixou de ser explicado sobre o $I H_{i{ }^{*}}$ Nesta modelagem, $\beta_{1}$ é o intercepto, $\beta_{2^{\prime}} \beta_{3^{\prime}} \beta_{4^{\prime}} \beta_{5^{\prime}} \beta_{6}$ e $\beta_{7}$ são os coeficientes do modelo e Ln se refere ao log natural das variáveis ajustadas. Além disso, i refere-se à unidade de observação e $\dagger$ à unidade de tempo.

Foram analisadas as três formulações econométricas alternativas: Pooled Regression, Modelo de Efeitos Aleatórios e Modelo de Efeitos Fixos, de modo a identificar mais informações e obter análise mais robusta das variações das unidades de observação ao longo do tempo (Wooldridge, 2010).

\section{RESULTADOS E DISCUSSÕES}

\subsection{ANÁLISE DAS ESTATÍSTICAS DESCRITIVAS}

Antes de iniciar a discussão sobre o modelo econométrico e seus resultados, é importante destacar algumas características das variáveis utilizadas, bem como fazer algumas considerações sobre resultados descritivos dos dados utilizados. Na Tabela 2, são apresentadas algumas estatísticas das variáveis para os anos de 1991, 2000 e 2010. O n representa o número de observações para cada UF. Cada dado coletado, em determinado município e em determinado ano, representa uma observação. 
Tabela 2

Estatística Descritiva das Variáveis (1991, 2000 e 2010)

\begin{tabular}{|c|c|c|c|c|c|}
\hline & $\begin{array}{c}\text { AMAZONAS } \\
(n=192)\end{array}$ & $\begin{array}{l}\text { DISTRITO FEDE- } \\
\quad \operatorname{RAL}(\mathrm{n}=3)\end{array}$ & $\begin{array}{c}\text { PERNAMBUCO } \\
(n=543)\end{array}$ & $\begin{array}{c}\text { PARANÁ }(n= \\
1.230)\end{array}$ & $\begin{array}{l}\text { SÃO PAULO (n } \\
\quad=1.908)\end{array}$ \\
\hline \multicolumn{6}{|c|}{ IDH (Índice) } \\
\hline Mín & 0,15 & 0,62 & 0,13 & 0,21 & 0,23 \\
\hline Méd & 0,43 & 0,72 & 0,46 & 0,58 & 0,63 \\
\hline Máx & 0,74 & 0,82 & 0,79 & 0,82 & 0,86 \\
\hline DP & 0,13 & 0,10 & 0,13 & 0,13 & 0,11 \\
\hline \multicolumn{6}{|c|}{ POPULAÇÃO (Em habitantes) } \\
\hline Mín & 4.464 & 1.553 .351 & 1.288 & 1.200 & 716 \\
\hline Méd & 43.390 & 2.035 .740 & 43.054 & 23.418 & 56.745 \\
\hline Máx & 1.793 .443 & 2.543 .802 & 1.524 .313 & 1.740 .948 & 11.172 .609 \\
\hline DP & 175.890 & 495.724 & 118.006 & 84.833 & 418.641 \\
\hline \multicolumn{6}{|c|}{ ENERGIA ELÉTRICA (\% de domicílios) } \\
\hline Mín & 12,9 & 98,3 & 14,0 & 26,6 & 41,6 \\
\hline Méd & 65,8 & 99,3 & 84,9 & 93,6 & 98,3 \\
\hline Máx & 100,0 & 99,9 & 100,0 & 100,0 & 100,0 \\
\hline DP & 20,9 & 0,9 & 19,8 & 11,1 & 4,6 \\
\hline \multicolumn{6}{|c|}{ SANEAMENTO (\% de domicílios) } \\
\hline Mín & 0,4 & 82,3 & 2,3 & 6,8 & 22,1 \\
\hline Méd & 24,3 & 90,3 & 47,4 & 80,6 & 92,7 \\
\hline Máx & 99,8 & 96,0 & 98,7 & 100,0 & 100,0 \\
\hline DP & 21,4 & 7,1 & 22,3 & 19,9 & 9,4 \\
\hline \multicolumn{6}{|c|}{ RENDA (R\$, per capta) } \\
\hline Mín & 69,0 & 916,0 & 39,5 & 77,3 & 142,8 \\
\hline Méd & 206,1 & $1.276,9$ & 215,6 & 426,3 & 563,4 \\
\hline Máx & 790,3 & $1.715,1$ & $1.144,3$ & $1.581,0$ & $2.043,7$ \\
\hline DP & 104,3 & 405,1 & 120,3 & 190,6 & 215,1 \\
\hline \multicolumn{6}{|c|}{ LOCALIZAÇÃO (Em km) } \\
\hline Mín & 0,0 & 0,0 & 0,0 & 0,0 & 0,0 \\
\hline Méd & 501,9 & 0,0 & 211,1 & 316,9 & 295,7 \\
\hline Máx & 1476,3 & 0,0 & 676,6 & 534,6 & 666,9 \\
\hline DP & 379,4 & 0,0 & 170,2 & 129,1 & 166,6 \\
\hline \multicolumn{6}{|c|}{ VIOLÊNCIA (Homicídios por 100 mil hab) } \\
\hline Mín & 0,0 & 30,9 & 0,0 & 0,0 & 0,0 \\
\hline Méd & 6,9 & 34,5 & 28,8 & 14,8 & 11,1 \\
\hline Máx & 47,2 & 38,3 & 135,8 & 145,7 & 124,9 \\
\hline DP & 9,4 & 3,7 & 22,1 & 18,5 & 16,0 \\
\hline
\end{tabular}

Fonte: Elaboração própria.

O IDH apresenta seu menor valor em um município de Pernambuco $(0,13)$ e seu maior valor em um município de São Paulo $(0,86)$, tendo sido percebido que a média mais alta está no Distrito Federal $(0,82)$. Percebe-se que para um índice que varia entre 0 e 1 , o desvio 
padrão identificado (entre 0,10 e 0,13) mostra-se relevante e oportuniza para a presente pesquisa a identificação dos fatores que explicam essas variações.

Em relação à população, o município menos populoso encontra-se em São Paulo, com apenas 716 habitantes. São Paulo também é a UF do município mais populoso, com cerca de 11 milhões de habitantes. Chama atenção o desvio padrão populacional no Paraná (84.833), que indica que os municípios nesta UF possuem menor variação populacional comparada.

No que tange à variável referente ao percentual de domicílios com energia elétrica, é importante salientar que em determinado município do Amazonas, apenas $12,9 \%$ dos domicílios possuem energia elétrica, enquanto a média em São Paulo é de 98,3\%. Trata-se de condição de infraestrutura com amplo acesso em São Paulo, Paraná e Distrito Federal, mas que ainda requer avanços em Pernambuco e Amazonas.

Já no que concerne às condições de saneamento, verifica-se importante diferença. Enquanto a média dos municípios do Amazonas e Pernambuco, com água encanada e banheiro, é de $24,3 \%$ e $47,4 \%$, respectivamente, em São Paulo esta média é superior a $92 \%$. Os dados mostram que as condições de saneamento nos municípios das regiões Norte e Nordeste, em média, são bastante inferiores às das regiões Sul, Sudeste e Centro-Oeste.

Em relação à variável renda, a média do Distrito Federal $(R \$ 1.276,90)$ é muito superior aos demais municípios, superando em mais de duas vezes a média de São Paulo $(R \$ 563,40)$ e em mais de seis vezes a do Amazonas ( $R \$ 206,10)$. Com todas as outras variáveis constantes, a alta renda no Distrito Federal pode explicar seu alto IDH.

A distância média dos municípios para a capital, representada pela variável localização, é maior no Amazonas (501,9km), sendo que nessa UF também se encontra o município mais distante da capital de sua unidade da federação $(1.476,3 \mathrm{~km})$. Considerando que o território amazonense é o maior do País, o resultado não surpreendeu.

Os números relativos à violência também precisam ser salientados. $O$ município pesquisado com maior número de homicídios a cada 100 mil habitantes é encontrado no Paraná (145,7), número quase quatro vezes maior do que o pior índice do Distrito Federal $(38,3)$. Entretanto, é sabido que dados sobre violência precisam ser minuciosamente avaliados, tendo em vista ser um tema de difícil abordagem e de resultados pouco confiáveis, devido à complexidade na busca por informações fidedignas (Oliveira \& Lopes, 2019).

\subsection{ANÁLISE DOS RESULTADOS DO MODELO ECONOMÉTRICO}

Inicialmente, utilizou-se o modelo Pooled Regression que envolve o empilhamento de dados, a utilização de regressão por mínimos quadrados ordinários - MQO e a premissa de comportamento não-heterogêneo das unidades de observação ao longo do tempo 
(Wooldridge, 2010). Os resultados (Tabela 3) mostraram significância estatística a 1\% para as variáveis, bem como o resultado do teste $F(p$-valor $=0$ ), que indicou significância global do modelo.

Tabela 3

Método Pooled Regression

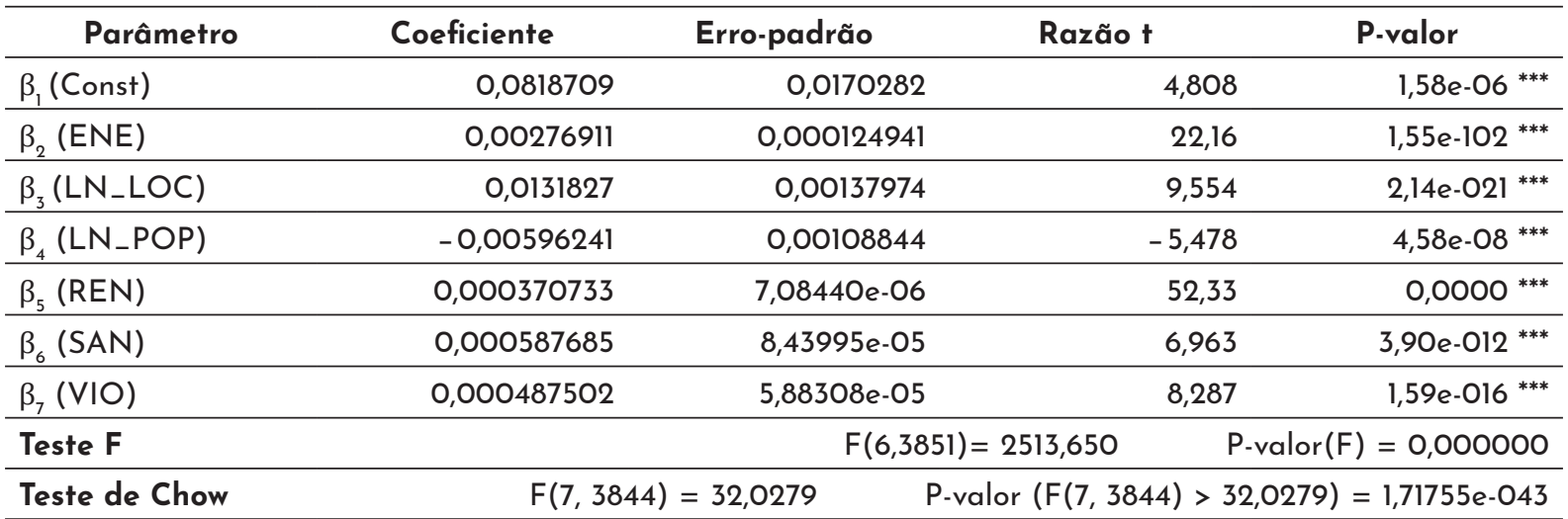

Fonte: Elaboração própria.

Para identificar se o Pooled Regression é adequado para a análise, foi necessário realizar teste para diferenciar interceptos. Foi utilizado o Teste de Chow, no qual a hipótese nula é a de que os grupos têm intercepto comum (Thursby, 1985). Conforme mostra a Tabela 3, os resultados do Teste de Chow mostraram que a probabilidade de erro Tipo 1 é quase nula. Em outras palavras, a probabilidade de se rejeitar a hipótese $\mathrm{H}_{0}$ quando ela não poderia ser rejeitada é quase nula ( $p$-valor $=1,71755 e-043$ ). Logo, o método Pooled Regression foi descartado e promoveu-se análise dos dados em painel, com efeitos fixos ou efeitos aleatórios.

Assim, realizou-se a análise dos dados em painel, pelo método dos efeitos aleatórios e, novamente, os resultados indicaram significância a 1\% para todas as variáveis e os mesmos coeficientes para os parâmetros, conforme mostra a Tabela 4.

Tabela 4

Método Efeitos Aleatórios

\begin{tabular}{lrrrr}
\hline Parâmetro & Coeficiente & Erro-padrão & Razão $t$ & P-valor \\
\hline$\beta_{1}$ (Const) & 0,0818709 & 0,0170282 & 4,808 & $1,52 e-06^{* * *}$ \\
\hline$\beta_{2}$ (ENE) & 0,00276911 & 0,000124941 & 22,16 & 7,78 e-109 $9^{* * *}$ \\
\hline$\beta_{3}$ (LN_LOC) & 0,0131827 & 0,00137974 & 9,554 & $1,24 e-021^{* * *}$ \\
\hline$\beta_{4}$ (LN_POP) & $-0,00596241$ & 0,00108844 & $-5,478$ & 4,30 e-08 $8^{* * *}$ \\
\hline$\beta_{5}$ (REN) & 0,000370733 & 7,08440 e-06 & 52,3 & $0,0000^{* * *}$ \\
\hline$\beta_{6}$ (SAN) & 0,000587685 & $8,43995 e-05$ & 6,963 & 3,33 e-012 $2^{* * *}$ \\
\hline$\beta_{7}$ (VIO) & 0,000487502 & $5,88308 e-05$ & 8,287 & $1,17 e-016^{* * *}$ \\
\hline
\end{tabular}

Teste de Hausman Qui-quadrado(5) $=5020,4 \quad$ P-valor $=0$

Fonte: Elaboração própria. 
No entanto, pelo Teste de Hausman é possível observar que o p-valor é nulo. Ou seja, a probabilidade de erro tipo 1 - rejeitar $\mathrm{H}_{0}$ quando não deveria ser rejeitada - também é nula. Como $\mathrm{H}_{\mathrm{o}}$ indica que os efeitos são aleatórios, o método deve ser descartado para se utilizar os efeitos fixos (Thursby, 1985).

Assim, foi utilizado o método dos efeitos fixos por meio dos mínimos quadrados ponderados - MQP (Tabela 5). O uso do MQP objetivou corrigir a heteroscedasticidade identificada nos resíduos por meio do Teste de Wald. Por meio desse teste verificou-se que os resíduos eram heteroscedásticos e que precisavam ser corrigidos - Qui-quadrado(1287) = 5,79335e+025; p-valor $=0$ (Wooldridge, 2010).

Tabela 5

Método Efeitos Fixos (Mínimos Quadrados Ponderados)

\begin{tabular}{|c|c|c|c|c|}
\hline Parâmetro & Coeficiente & Erro-padrão & & P-valor \\
\hline$\beta_{1}$ (Const) & 0,116673 & 0,0115938 & 10,06 & $1,56 e-023^{* * *}$ \\
\hline$\beta_{2}(E N E)$ & 0,00303153 & 9,1040le-05 & 33,30 & $6,88 e-214^{* * *}$ \\
\hline$\beta_{3}\left(L N_{-} L O C\right)$ & 0,0107087 & 0,00104736 & 10,22 & $3,14 \mathrm{e}-024^{* * *}$ \\
\hline$\beta_{4}\left(L N_{-} P O P\right)$ & $-0,0111295$ & 0,000779489 & $-14,28$ & $4,18 e-045^{* * *}$ \\
\hline$\beta_{5}(\mathrm{REN})$ & 0,000421519 & $5,71004 e-06$ & 73,82 & $0,0000^{* * *}$ \\
\hline$\beta_{6}(\mathrm{SAN})$ & 0,000339468 & $6,73427 e-05$ & 5,041 & $4,85 e-07^{* * *}$ \\
\hline$\beta_{7}(\mathrm{VIO})$ & 0,000618605 & $4,27833 e-05$ & 14,46 & $3,49 e-046^{* * *}$ \\
\hline Teste F & & \multicolumn{2}{|l|}{$F(6,3851)=5388,149$} & $=0,000000$ \\
\hline R-quadrado & & & & 0,893560 \\
\hline
\end{tabular}

Fonte: Elaboração própria.

Assim, tem-se os resultados da análise de dados em painel por todas as formulações econométricas realizadas (Tabela 6). Os resultados mais ajustados relacionam-se ao método de efeitos fixos por mínimos quadrados ponderados.

Tabela 6

Parâmetros calculados

\begin{tabular}{|c|c|c|c|c|c|c|}
\hline Parâmetro & Pooled Regressior & & Efeitos Aleatórios & & Efeitos Fixos (MQP & \\
\hline$\beta_{1}$ & 0,0818709 & ${ }^{* * *}$ & 0,0818709 & ${ }^{* * *}$ & 0,116673 & *** \\
\hline $\mathrm{B}_{2}$ (Energia) & 0,00276911 & ${ }^{* * *}$ & 0,00276911 & ${ }^{* * *}$ & 0,00303153 & $* * *$ \\
\hline $\mathrm{B}_{3}$ (Localização) & 0,0131827 & ${ }^{* * *}$ & 0,0131827 & ${ }^{* * *}$ & 0,0107087 & $* * *$ \\
\hline $\mathrm{B}_{4}$ (População) & $-0,00596241$ & $* * *$ & $-0,00596241$ & $* * *$ & $-0,0111295$ & $* * *$ \\
\hline $\mathrm{B}_{5}$ (Renda) & 0,000370733 & $* * *$ & 0,000370733 & $* * *$ & 0,000421519 & $* * *$ \\
\hline $\mathrm{B}_{6}$ (Saneamento) & 0,000587685 & ${ }^{* * *}$ & 0,000587685 & ${ }^{* * *}$ & 0,000339468 & $* * *$ \\
\hline $\mathrm{B}_{7}$ (Violência) & 0,000487502 & $* * *$ & 0,000487502 & $* * *$ & 0,000618605 & $* * *$ \\
\hline
\end{tabular}

Fonte: Elaboração própria. 
Verifica-se que o modelo possui significância global e seis variáveis significantes a $1 \%$, para explicar a relação funcional entre variáveis socioeconômicas e o IDH. Em outras palavras, energia elétrica, localização, população, renda, saneamento e violência mostraramse como fatores explicativos do IDH.

Pela literatura suporte, esperava-se relação funcional positiva entre a presença de energia elétrica em domicílios e o IDH. Estudo conduzido por Ferreira e Malliagros (1998) já indicava forte relação entre a adoção de políticas públicas de investimento no setor de energia elétrica e o nível de renda no Brasil. Em complemento a esta análise, Bispo (2009) confirmou que a presença de energia elétrica em domicílios foi um fator explicativo do IDH no Estado de Roraima. Os resultados deste trabalho confirmaram essa expectativa. A melhoria nas condições de energia elétrica tende a implicar em melhoria no IDH nos municípios. A maior presença de energia elétrica proporciona melhores condições de produção de renda, maior acesso à conhecimento, educação, saúde, entre outros elementos relacionados ao desenvolvimento humano.

A partir de estudos anteriores, esperava-se que a relação funcional entre a localização do município e o IDH poderia ser negativa ou positiva. Em estudo realizado em Alagoas, Ângelo et al. (2009, p. 44) indica que "quanto mais longe da capital, não somente se tem menos acesso aos serviços públicos, mas também há menos possibilidades de o indivíduo se desenvolver tanto no âmbito profissional quanto no pessoal". Por outro lado, estudo realizado no Paraná, identificou que municípios distantes da capital relacionaram-se com maiores níveis de IDH (Scarpin \& Slomski, 2007). O resultado da presente pesquisa indicou forte relação funcional positiva entre a distância à capital e o IDH, confirmando o achado de Scarpin e Slomski (2007) e refutando o argumento de Ângelo et al. (2009). A relação funcional positiva encontrada pode ser explicada pela forte presença do estado de São Paulo na amostra. É sabido que o interior paulista é muito desenvolvido e que cidades afastadas da capital possuem elevado IDH. A localização mostra-se como um fator explicativo de IDH, mas é necessário compreender melhor a situação social e econômica de cada região para se projetar impactos positivos ou negativos sobre oIDH.

Em relação a população dos municípios, a literatura suporte analisada prevê relação funcional negativa entre esta variável e o IDH. É possível depreender que localidades com elevado tamanho populacional tenham problemas em seus níveis de emprego e outros indicadores sociais, o que pode significar que aumento na população diminui tanto a qualidade de vida como o desenvolvimento humano (Angelo et al., 2009; Matta et al., 2016). Os resultados encontrados confirmaram as expectativas. Com o crescimento da população, espera-se um decréscimo no IDH, mantendo-se as demais variáveis constantes. Nessa linha, políticas públicas de contenção do crescimento populacional podem redundar no aumento da qualidade de vida da população. 
O fator renda tem robusta literatura de suporte que indique sua relação funcional positiva com o IDH. Maiores níveis de renda relacionam-se com melhores condições de planejamento familiar para satisfação das necessidades humanas, acesso a transporte, habitação, saneamento e outros elementos que favorecem melhores condições de vida (Matta et al., 2016). Os resultados desta pesquisa corroboraram o indicado pela literatura ao mostrar que esta relação funcional ocorre em sinal positivo. Quanto maior a renda, maior o desenvolvimento humano. Este resultado também foi encontrado por estudos conduzidos por Jalil e Kamaruddin (2018) e Lestari e Sanar (2018). Além de significar uma medida de bem-estar financeiro, a renda também pode ser considerada um fator de melhoria do IDH.

Situação semelhante à renda foi verificada na variável saneamento. Os resultados confirmaram expectativa de relação funcional positiva. Melhores condições sanitárias banheiro e água encanada nos domicílios, por exemplo - relacionam-se com aumento no IDH. Este resultado confirma os achados de ljaz (2018) e de Scarpin e Slomski (2007), segundo os quais o aperfeiçoamento nas condições de saneamento são importantes para o desenvolvimento (ljaz, 2018).

O resultado referente à variável de violência não permitiu clara conclusão nesta pesquisa. É intuitivo que o nível de homicídios esteja negativamente relacionado ao IDH. No entanto, isso não foi verificado pelos resultados encontrados na pesquisa. Com o aumento na taxa de homicídios, tudo mais constante, esperava-se um aumento médio no IDH dos municípios. Relação funcional positiva entre violência e IDH também foi encontrada por Angelo et al. (2009). Trata-se, portanto, de questão que precisa ser melhor investigada em estudos futuros. A utilização de diferentes fontes de dados pode jogar luz sobre estes resultados, considerando-se, como dito acima, que não é fácil encontrar dados confiáveis sobre o tema da violência (Oliveira \& Lopes, 2019).

\section{CONCLUSÕES}

$O$ presente trabalho teve como objetivo investigar quais fatores explicam o IDH de municípios brasileiros, de modo a contribuir para que políticas públicas possam ser promovidas com foco no aumento no desenvolvimento humano.

A partir de literatura suporte e com a análise de dados em painel de 1.292 municípios brasileiros - nos anos de 1991, 2000 e 2010 - foi possível identificar seis variáveis socioeconômicas explicativas do IDH: energia elétrica, localização, população, renda, saneamento e violência. O modelo mostrou significância global e todas as variáveis mostraram-se significantes a 1\%, o que permitiu a confirmação de suas relações funcionais com o IDH.

O investimento em políticas públicas que promovam melhores condições de infraestrutura (casos da energia elétrica e do saneamento) e que promovam melhores níveis 
de renda para a população, tendem a acarretar mais elevados níveis de desenvolvimento humano nos municípios. Além disso, políticas públicas voltadas a conter aumentos populacionais também tendem a promover o desenvolvimento. $O$ fator localização mostrouse explicativo do IDH, mas a direção em que esta influência ocorre depende das condições sociais e econômicas de cada região. Em algumas regiões, os municípios distantes da capital relacionam-se com maior IDH; em outras regiões, relacionam-se com menor IDH. Quanto ao fator violência, identificou-se que ele é um fator explicativo do IDH, mas a relação funcional positiva, que relaciona maiores níveis de violência à um maior nível de IDH, não foi intuitiva. Assim, avançar nos estudos que identifiquem a relação da violência com o IDH é uma importante agenda de pesquisa para trabalhos futuros.

Do ponto de vista teórico, a presente pesquisa ofereceu método e variáveis que podem contribuir com a identificação de fatores explicativos para o IDH. Foi possível avançar em relação a estudos anteriores (Jalil \& Kamaruddin, 2018; Matta et al., 2016; Mattei et al., 2018; Scarpin \& Slomski, 2007) que objetivavam identificar a relação funcional de fatores socioeconômicos e IDH por meio do uso do método de regressão. Novas variáveis e fontes de dados foram utilizadas, com novos resultados alcançados, de modo a contribuir para o debate teórico sobre o tema.

Do ponto de vista prático, a pesquisa indicou caminhos para a promoção de políticas públicas que poderão melhorar os níveis de desenvolvimento humano nos municípios brasileiros. Os resultados desta pesquisa sugerem que gestores públicos podem realizar investimentos em melhores condições de energia elétrica, saneamento e renda com efeitos positivos sobre o desenvolvimento humano da população. Além disso, medidas para conter crescimentos populacionais podem ser úteis para promoção do IDH. Além disso, os resultados encontrados sugerem que gestores públicos adotem ferramentas de monitoramento dos fatores e variáveis propostos, de modo a antecipar melhorias que possam repercutir no desenvolvimento humano de seus municípios.

A principal limitação desta pesquisa envolveu o uso de dados temporais. A utilização de painel com os dados apenas dos anos de 1991, 2000 e 2010 foi uma restrição imposta pelo fato de que apenas nestes anos houve apuração do IDH pelo PNUD. Assim, é indicado que novos estudos sejam realizados, na medida em que sejam feitas atualizações do IDH pelo PNUD.

\section{REFERENNCIAS}


Angelo, L. de C., Ramos, F. de S., \& De Souza, H. R. (2009). Fatores Explicativos do Índice de Desenvolvimento Humano (IDH) para os municípios de Alagoas. Economia Política Do Desenvolvimento, 1(6), 31-47.

Atlas Brasil. (2021). Acervo. http://www.atlasbrasil.org.br/acervo/atlas.

Bilbao-Ubillos, J. (2013). Another approach to measuring human development: the composite dynamic human development index. Social Indicators Research, 111(2), 473-484. https://doi.org/10.1007/s11205-012-0015-y.

Bispo, R. de S. (2009). IDH de Roraima: condicionantes e especificidades. Universidade Federal do Rio Grande do Sul.

Bresser-Pereira, L. C. (2003). Desenvolvimento e crise no Brasil: história, economia e política de Getúlio Vargas a Lula. Editora 34.

Castelar, A. (2009). O Brasil precisa de uma estratégia de desenvolvimento? In J. Sicsú, \& A. Castelar (Eds.), Sociedade e economia: estratégias de crescimento e desenvolvimento (pp. 9-12). Ipea.

Dias, R., \& Matos, F. (2012). Políticas públicas: princípios, propósitos e processos (1 $1^{a}$ ed.). Editora Atlas.

Fadilah, A., Ananda, C. F., \& Kaluge, D. (2018). A panel approach : how does government expenditure influence human development index? Jurnal Ekonomi Dan Studi Pembangunan, 10(2), 130-139.

Ferreira, P. C., \& Malliagros, T. G. (1998). Impactos produtivos da infra-estrutura no Brasil 1950/95. Pesquisa e Planejamento Econômico (PPE), 28(2), 315-338.

Fukuda-Parr, S. (2003). The human development paradigm: Operationalizing Sen's ideas on capabilities. Feminist Economics, 9(2-3), 301-317. https://doi.org/10.1080/135457002200 0077980 .

Gelman, A., \& Hill, J. (2007). Data analysis using regression and multilevel/hierarchical models. Cambridge University Press.

Guimarães, J. R. S., \& Jannuzzi, P. de M. (2005). IDH, indicadores sintéticos e suas aplicações em políticas públicas: uma análise crítica. Revista Brasileira de Estudos Urbanos e Regionais, 7(1), 73. https://doi.org/10.22296/2317-1529.2005v7nlp73.

Höfling, E. de M. (2001). Estado e políticas (públicas) sociais. Cadernos CEDES, 21(55), 30 41. https://doi.org/10.1590/S0101-32622001000300003.

ljaz, M. (2018). Does poverty reduction cause economic development. Sukkur IBA Journal of Economics and Finance, 2(1), 36. https://doi.org/10.30537/sijef.v2il.200.

Ipeadata. (2021). Conjunto de dados. https://dados.gov.br/dataset/ipeadata. 
Jalil, S. A., \& Kamaruddin, M. N. (2018). Examining the relationship between human development index and socio-economic variables: a panel data analysis. 3(2), 37-44.

Khair, A. (2009). Caminhos para o desenvolvimento: uma visão estratégica. In J. Sicsú, \& A. Castelar (Eds.), Sociedade e economia: estratégias de crescimento e desenvolvimento (pp. 59-70). Ipea.

Lestari, W. W., \& Sanar, V. E. (2018). Analysis indicator of factors affecting human. 2(1), 1118.

Machado, J. G. R., \& Pamplona, J. B. (2008). A ONU e o desenvolvimento econômico: uma interpretação das bases teóricas da atuação do PNUD. Economia e Sociedade, 17(1), 53 84. https://doi.org/10.1590/s0104-06182008000100003.

Marinho, E., \& Neto, P. de M. J. (1999). Gastos públicos e condições de vida nos municípios do estado do Ceará. Revista de Administração Pública, 33(3), 139-160.

Martins, A. R. P., Ferraz, F. T., \& Costa, M. M. da. (2006). Sustentabilidade ambiental como nova dimensão do índice de desenvolvimento humano dos países. Revista do BNDES, 13(26), 139-162.

Matta, I., Ferreira, M., Cotta, R., \& Siqueira-Batista, R. (2016). Gestão da saúde pública: análise sobre os fatores condicionantes do desenvolvimento humano. Revista de Gestão em Sistemas de Saúde, 5(1), 150-28. https://doi.org/10.5585/rgss.v5il.200.

Mattei, T. F., Bezerra, F. M., \& Mello, G. R. de. (2018). Despesas públicas e o nível de desenvolvimento humano dos estados brasileiros: uma análise do IDHM 2000 e 2010. RACE - Revista de Administração, Contabilidade e Economia, 17(1), 29. https://doi.org/10.18593/ race.v17il.10296.

Neumayer, E. (2001). The human development index and sustainability - a constructive proposal. Ecological Economics, 39(1), 101-114. https://doi.org/10.1016/S09218009(01)00201-4.

Oliveira, W. F. (2013). Índice de desenvolvimento humano e pegada ecológica: uma proposta de integração. ANPEC, 1-20.

Oliveira, A., \& Lopes, S. (2019). Revista de Saúde Coletiva da UEFS. 9, 186-192. https://doi. org/10.13102/rscdauefs.v9.3685.

Programa das Nações Unidas para o Desenvolvimento. (1990). Human Development Report 1990.

Programa das Nações Unidas para o Desenvolvimento. (2010). Relatório de Desenvolvimento Humano. https://www.undp.org/content/dam/brazil/docs/RelatoriosDesenvolvimento/undp-br-PNUD_HDR_2010.pdf.

Sagar, A. D., \& Najam, A. (1998). The human development index: a critical review. Ecological Economics, 25(3), 249-264. https://doi.org/10.1016/S0921-8009(97)00168-7. 
Scarpin, J. E., \& Slomski, V. (2007). Estudo dos fatores condicionantes do índice de desenvolvimento humano nos municípios do estado do Paraná: instrumento de controladoria para a tomada de decisões na gestão governamental. Revista de Administração Pública, $4 l$ (5), 909-933. https://doi.org/10.1590/s0034-76122007000500006.

Secchi, L. (2015). Políticas públicas: conceitos, esquemas de análise, casos práticos ( $2^{a}$ ed.). Editora Trilha.

Sicsú, J. (2009). A construção de uma estratégia de desenvolvimento. In J. Sicsú, \& A. Castelar (Eds.). Sociedade e economia: estratégias de crescimento e desenvolvimento (pp. 19-27). Ipea.

Souza, C. (2006). Políticas públicas: uma revisão da literatura. Sociologias, 16, 20-45. https://doi.org/10.1590/S1517-45222006000200003.

Thursby, J. G. (1985). The relationship among the specification tests of Hausman, ramsey, and chow. Journal of the American Statistical Association, 80(392), 926-928. https://doi. org/10.1080/01621459.1985.10478205.

Trabold-Nübler, H. (1991). The human development index-A new development indicator? Intereconomics, 26(5), 236-243. https://doi.org/10.1007/BF02928996.

Wooldridge, J. M. (2010). Econometric analysis of cross section and panel data. The MIT Press. https://doi.org/10.1007/s12053-016-9491-2. 
